\title{
II.
}

\section{Zur Lehre von der Erb'schen Krankheit}

(Myasthenia psendoparalytica, asthenische Bulbärparalyse).

Von

\section{Dr. 0. Giese und Prof. Fr. Schultze \\ in Bonn.}

Wenn die Nomenclatur eines noch ziemlich jungen Krankheitsbildes eine derartige Vielseitigkeit aufweist, wie dies bei der asthenischen Bulbärparalyse (Myasthenia pseudoparalytica, schwere allgemeine Myasthenie, Bulbärparalyse ohne anatomischen Befund, Bulbärparalyse Typus Erb-Goldflam, Erb'sche Krankheit) der Fall ist, so spricht schon dieser Umstand dafür, dass die Anschauungen der Autoren in Bezug auf das Wesen und die Klassificirung dieses Leidens keine einheitlichen sind. Zwar umfasst die Literatur bereits einige 50 einschlägigen Beobachtungen, wodurch wenigstens die Symptomatologie dieser interessanten Erkrankung eine ziemlich gute $\mathrm{Ab}$ grenzung erfahren hat, dagegen sind unsere Kenntnisse über die Aetiologie und den anatomischen Sitz des Processes noch ganz unbefriedigende. Vermögen auch die folgenden Zeilen nicht dies Dunkel zu lichten, so dürfte doch der zu beschreibende Fall um so mehr Interesse bieten, als durch ihn die bisher noch ziemlich beschränkte Anzahl von Autopsien um einen Befund vermehrt werden konnte.

\section{Krankengeschichte (Dr. 0. Giese).}

Frau V. aus Ü., 24 J. alt, wurde am 2. Sept. 99 in die hiesige medicin. Klinik aufgenommen durch gütige Vermittelung des Herrn Dr. W $\theta$ ber, Nervenarzt in Dortmund, dessen Hülfe die Kranke nachgesucht hatte.

Dr. Weber konnte am 1. Sept. folgenden Krankheitsbefund erheben, auf Grund dessen er auch bereits die Diagnose "Myasthenie" stellte:

Blasses, elendes Aussehen der Kranken. Sprechen deutlich, aber sehr mühsam; dabei starke Luftverschwendung. Starke Ptosis rechts. Lidspalte rechts etwa $4 \mathrm{~mm}$ weit, kann auf ca. $6 \mathrm{~mm}$ erweitert werden. $\mathrm{Pu}$ pillen mittelweit, gleich, prompt reagirend. Rechts Rectus internus ganz gelähmt, Rectus externus etwas paretisch. Augenschluss beiderseits unmöglich. Sonst Facialisgebiet ibberall gleichgut innervirbar, aber ohne Kraft; der Innervationstonus lässt schnell nach. Pfeifen und Blasen gelingt nicht, auch Husten nicht, so dass die Expectoration - es besteht 
mässige Bronchitis - hochgradig erschwert ist. Das Gaumensegel ist ganz gelähmt; die Zunge ist schwer beweglich, insofern als ihre Bewegungen nur langsam ansgeführt werden können und angenscheinlich grosse Kraftanstrengung erfordern; dann aber gelingen ausgiebige Bewegungen nach allen Richtungen. Fibrilläre Zuckungen sind nirgends vorhanden, die Zunge zittert nur in toto etwas (rhythmische Bewegung vorand rückwärts). - Musculatur der Extremitäten gut entwickelt, aber ebenfalls von herabgeminderter Kraft. Arme nur geringer Kraftanstrengung fähig, ermüden schnell. Keine fibrillären Zuckungen. Am Halse und am Rumpfe keine deutliche Parese. Auch die Beinmusculatur sehr schwach, links mehr als rechts. Am ausgesprochensten ist die Schwäche im linken Iliopsoas: das 1. Bein kann nur bis zum halben rechten Winkel gehoben werden.

Patellarreflexe lebhaft, nicht abnorm. An den Armen sind die Reflexe schwach. Kieferreflex fehlt. Sensibilität überall intact. Elektrische Untersuchung: Facialis spricht erst bei $2^{1 / 2}$ M.-A. eben an, deutlichere Zuckungen erst bei 4 M.-A.; keine Entartungsreaction. Nach etwa 20 Wendungen wurden die Zuckungen deutlich schwächer. Auch faradisch ist die Erregbarkeit herabgesetzt; ein Schwächerwerden der Contractionen hierbei nicht zu constatiren. -

Aus der Vorgeschichte der Kranken und aus dem ausführlichen Krankenjournal während ihres 6 wöchentlichen Anfenthaltes in der Klinik ist Folgendes hervorzuheben:

Die Kranke stammt von gesunden Eltern ab, die beide noch am Leben sind. Nervenkrankheiten wurden bisher nicht in der Familie beobachtet, nur eine Tante war vor 9 Jahren wegen cerebraler Lnes in hiesiger Klinik in Behandlung, ist aber jetzt gesund. Von 5 Geschwistern der Patientin starb ein Kind an Krämpfen, eine Schwester an Typhus, 3 Brüder leben und sind gesund. Die Kranke selbst will stets gesund gewesen sein, nur litt sie znweilen an Kopfschmerzen, die mit Magenbeschwerden einhergingen. Die Menses traten mit 16 Jahren ein, waren stets regelmässig. - Im Alter von 21 Jahren hat sich die Kranke verheirathet, sie hat 2 gesunde Kinder; die Geburten verliefen normal.

Anfang Mai 1899 begann die Erkrankung mit einem 14 Tage anhaltenden Schnupfen und Husten (Influenza?); zugleich bestand zeitweise halbseitiger Kopfschmerz, meist rechts, verbunden mit Uebelkeit. Zur selben Zeit trat auch schon etwa 8-14 Tage lang Doppelt. sehen auf, das durch Elektrisiren besser geworden sein soll. - Ende Mai bemerkte die Kranke, dass ihr das Schlucken besonders von Flüssigkeiten schwerer fiel, indem diese leicht durch die Nase zurückkamen. Etwa 14 Tage später konnte Patientin zeitweise schlecht sprechen, besonders wenn sie einige Zeit lang gegessen hatte; dann trat auch Müdigkeit in der rechten Kiefergegend ein. Die Zunge war schwer bewegbar; die Bissen konnten nicht von einer Seite auf die andere geschoben werden. Diese Beschwerden nahmen allmählich za, wurden jedoch in den letzten 14 Tagen wieder bedeutend geringer. - Anch das Doppeltsehen stellte sich Mitte Juni wieder ein und blieb seitdem in wechselnder Stärke bestehen. Vor 5 Wochen nahm die Kranke wahr, dass das rechte Angenlid ,herunterfiel" und nicht mehr gehoben werden konnte. - Seit derselben Zeit tritt ein- bis mehrmals täglich eine vorübergehende Erlahmung 
des 2. und 3. Fingers auf, manchmal anch des Daumens, und zwar beiderseits hauptsächlich, wenn die Kranke längere Zeit mit den Fingern gearbeitet hat; dabei soll auch Brennen im Verlauf des Medianus am Unterarm bestehen. Gleichzeitig wurden die Arme schwach und in noch höherem Grade das linke Bein, in dem die Schwäche jetzt eine dauernde und beständig im Zunehmen befindliche sein soll. - Auch die Bewegungen des Kopfes waren erschwert: etwa 8 Tage lang fiel er beim Sitzen nach vorn; das wurde dann besser, dafür konnte nun aber der Kopf beim Sitzen nicht mehr erhoben werden. - Schliesslich trat auch noch vor 8-14 Tagen eine völlige Aphonie auf, während die Stimme bis dahin laut tönend gewesen war.

Schmerzen oder Parästhesien waren mit Ausnahme des erwähnten „Brennens“ im Medianusgebiet nie vorhanden. Fieber, Erbrechen, Schwindel fehlten während der ganzen Krankheitsdauer, ebenso Ausfallerscheinungen von Seiten der Blase und des Mastdarmes. Die Kranke klagt jetzt hauptsächlich über Müdigkeit in allen Gliedern und anhaltende Schlaflosigkeit.

Status praesens. Die Kranke ist von kräftigem Körperban; die Musculatur im Allgemeinen ziemlich gut entwickelt, Fettpolster desgl. Hant und Schleimhänte blass. Keine Drüsenanschwellungen. Es besteht eine geringe Scoliose der Halswirbelsäule. Die linke Infraclaviculargrube ist anders configurirt als die rechte, wohl in Folge der stark hervorspringenden linken Querfortsätze der Halswirbel.

Sensorium frei. Psyche intact.

Auf dem r. Ange ist die Sehschärfe etwas herabgesetzt, sonst an den sensorischen Hirnnerven keine Ausfallerscheinungen. Pupillen gleichweit, von mittlerer Grösse, reagiren prompt anf Licht, Convergenz und Accomodation. Augenhintergrund normal. Augenbewegungen links frei, rechts bei Bewegungen nach oben und unten keine Störung, nach aussen und innen Einschränkung bis zur Hälfte der normalen Bewegungsfähigkeit. Kein Nystagmus. Das r. Augenlid hängtherunter, so dass es beim Geradeaussehen die Pupille fast ganz verdeckt. Links keine Ptosis. Augenschluss gelingt beiderseits sehr mangelhaft. Stirnrunzeln ebenfalls. Lidreflex von Cornea nnd Conjunctiva auslösbar, aber schwach. Orbicnlaris oris beiderseits paretisch, im Gebiet der unteren Facialisäste keine Störung, Kaumuskeln wirken kräftig. Zunge wird gerade hervorgestreckt, zittert nicht. Beim Schlucken keine Störung. Articnlation gut. Stimme aphonisch. Das Gaumensegel wird willkürlich rechts gar nicht gehoben, links eine Spur. Gaumenreflex spurweise auslösbar, auch der Würgreflex an der hinteren Rachenwand nur schwer auszulösen. Kinnreflex vorhanden. Im Bereiche der Hals- und Nackenmuskeln keine Störung. Beim Aufrichten des Rumpfes werden die linken langen Rückenmuskeln schlechter innervirt als die rechten. Innervation der Bauchmuskeln gut. Bauchreflex nur rechts einmal deutlich zu erzielen.

An den oberen Extremitäten besteht rechts eine lähmungsartige Schwäche aller Muskeln, im Gebiete des Radialis und Medianus stärker als im Ulnarisgebiet. Die Reflexe an den Armen sind schwach, aber dentlich; der Supinatorreflex ist rechts schwächer als links.

An den unteren Extremitäten ist der linke Iliopsoas paretisch, auch im Kniegelenk ist die Bengung links schwächer als rechts. Patellareflex von normaler Stärke, beiderseits gleich. Achillesreflex vorhanden, 
links schwächer. Plantareflex schwach und nicht jedesmal zu erzielen; er besteht in Plantarflexion der Zehen.

Nirgends deutliche Atrophie, keine fibrillären Zuckungen. Die Sensibilität ist überall und für alle Qualitäten normal. Keine Coordinationsstörungen. Weder im Bereiche der Hirnnerven, noch an den Extremitäten lässt sich eine sichere Ermüdbarkeit nachweisen. Prüfung des Händedruckes mit dem Dynamometer ergab folgende Zahlenreihen:

rechts: $32,32,32,20,30,30,17,27,40,37$

links: $36,40,32,42$

Laryngoskopischer Befund: Die Excursionen der Stimmbänder nach innen und aussen sehr gering; besonders ist die Abduction beiderseits minimal. Beim Intoniren kein völliger Schluss der Stimmbänder. Eine Differenz zwischen beiden Seiten nicht nachweisbar. An der Schleimhaut des Kehlkopfes keine Veränderungen.

Die elektrische Untersuchung ergiebt weder qnantitative noch qualitative Veränderungen; nur im Facialis besteht eine geringe Herabsetzung der Erregbarkeit für beide Stromarten. Myasthenische Reaction nicht nachweisbar. An Lungen und Herz kein abnormer Befund. Puls von mittlerer Stärke, nicht immer ganz regelmässig, aber ohne starke Irregularität; zeitweise mässig beschleunigt. Leber etwas gesenkt, Leberrand unterhalb des Rippenbogens palpabel. Urin frei von Eiweiss und Zucker.

Blutuntersuchung: Hämoglobingehalt 95 Proc., rothe Blutkörperchen 3,6 Millionen, weisse 10300 .

Ordination: Bettruhe, Jodkalium; leichte Faradisirung, später Eisen.

5. IX. Heute Morgen ist die Stimme etwas weniger aphonisch; auch die Ptosis rechts ist geringer.

7. IX. Athemgeräusch heute sehr oberflächlich und leise, vorn l. oben Giemen; seit gestern reichlicher Auswurf, keine Tuberkelbacillen enthaltend. Supinatorreflex heute links eher schwächer als rechts.

10. IX. Das Gaumensegel wird etwas besser gehoben, aber nur nach links. Kopfnicker leicht paretisch. Kraft in den Armen jetzt gut, in den Fingern vielleicht noch eine leichte Abschwächung beim Strecken der Grundphalangen. Anch das Spreizen der Finger geschieht mit ziemlich geringer Kraft. Iliopsoas links noch paretisch. Keine deutliche Ermüdbarkeit nachweisbar.

11. IX. Heute Morgen ist die Ptosis der rechten Augenlider fast ganz geschwunden; anch der Augenschluss ist vollständiger und fester. Die Stimme hat etwas Klang. Die rechte Nasolabialfalte in der Rnhe etwas weniger ausgeprägt als links. Kieferreflex heute nicht auslösbar.

12. IX. Lidschluss heute Morgen links vollstïndig, während rechts ein ganz kleiner Spalt bleibt. Nachdem die Kranke etwa 2 Minuten lang die Augen geschlossen gehalten, ist auch links der Bulbus etwa $2 \mathrm{~mm}$ breit sichtbar; zugleich treten dann in beiden Augenlidern fibrilläre Zuckungen auf. Die anfangs kaum nachweisbare Ptosis am r. Ange wird, wenn man sich einige Minuten mit den Augen der Kranken beschäftigt, sehr deutlich: die Pupille ist jetzt zum Theil vom oberen Lid bedeckt. Die Wirkung der Sternocleidomastoidei geschieht zunächst mit ziemlich guter Kraft; lässt man die Kranke einige Zeit mit dem Kopf gegen die unter das Kinn gehaltene Hand drücken, so erlahmt die Kraft nach 25 Secunden und 
nach einigen weiteren Secunden sinkt der Kopf zurück. Elektrisch keine Ermüdbarkeit nachznweisen. Im $r$. Iliopsoas ist die Kraft annähernd normal, links bedeutend geringer, doch wird das linke Bein zunächst leidlich gut gehoben; bei wiederholtem Versuch wird die Kraft immer geringer, so dass nach 15maliger Wiederholung schon der leiseste Druck auf den Oberschenkel nicht mehr überwanden werden kann. Nach kurzer Ruhepause kann die Kranke das Bein wieder bis etwa zum halben rechtenWinkel erheben, doch kaum 10 Secunden frei halten, dann fällt es schlaff herunter.

14. IX. Hente ist, zugleich mit dem Einsetzen der Menses, eine dentliche Verschlimmerung zu constatiren. Die Athmung ist beschleunigt (etwa 30 i. d. Min.) und geschieht unter Anspannung der inspiratorischen Hülfsmuskeln. Die Zwerchfellathmung scheint gestört: Bei tiefer Inspiration Einziehung des Epigastrium; das Abdomen kann mit grosser Anstrengung nur ruckweise ein wenig vorgewölbt werden. Auch die Exspiration ist kraftlos, Husten ganz unmöglich; es besteht lautes Trachealrasseln. - Kraft der Sternocleidomastoidei sehr gering. Das Aufrichten ans dem Rückenlager nur durch Aufstützen der Arme möglich. Im l. Iliopsoas ist die Ermüdbarkeit heute sehr dentlich: dass Bein kann nur etwa 15 mal gehoben werden. Auch die Adductoren des 1 . Beines wirken heute deutlich schwächer. - Das Schlucken ist ebenfalls wioder erschwert. Die Ptosis rechts ist stärker geworden; der Augenschluss beiderseits unvollkommen, besonders links bleibt ein mehrere mm breiter Spalt. Das Gaumensegel wird beim Versuch zu intoniren manchmal eine Spur nach links gehoben, manchmal garnicht. Gaumenreflex fehlt.

Händedruck beiderseits recht schwach.

Dynamometer: linke Hand: $20,20,15,15,11,10,9,10$

rechts: $20,20,20,20,18,18,14,11,11,14,9,9$

Ordination: Campher subcutan. Tinct. nuc. vomic. 3 mal 5 Tropfen.

17. IX. Das Allgemeinbefinden ist hente wieder viel besser. Respiration normal, Ptosis geringer, Augenschluss anch längere Zeit hindurch ziemlich kräftig. Gaumensegel wird viel besser gehoben, hauptsächlich nach links, doch auch rechts etwas. Miopsoas wirkt viel kräftiger, das 1. Bein kann auch längere Zeit erhoben gehalten werden.

In den folgenden Tagen macht die Besserung im Allgemeinen Fortschritte; besonders nimmt die Kraft im l. Bein ständig zu, so dass keine deutliche Ermïdbarkeit mehr beobachtet werden kann. Auch im Bereiche der meisten Hirnnerven macht sich die Neigung zur Besserung bemerkbar. Die Kranke giebt selbst an, dass sie empfinde, wie die Action der Stirnund Wangenmuskeln wieder freier werde. Objectiv erscheint das Runzeln der Stirn zwar möglich, aber nur in geringem Grade. Ptosis nur noch sehr gering. Beim Blick nach rechts bleibt das r. Auge noch etwas zurück; beim Blick nach rechts treten gleichnamige Doppelbilder auf, sobald die Blickrichtung etwa $30^{\circ}$ von der Mittellinie abweicht; auch bei der äussersten Einstellung nach links treten zeitweise gekreuzte Doppelbilder auf. Die Sprache hat znweilen Morgens etwas Klang, im Laufe des Tages wird sie wieder aphonisch. Die elektr. Erregbarkeit ist nirgends gestört; im Facialis sowohl vom Nerven, wie vom Muskel aus 1. Zuckung bei 1 M.-A.

29. IX. Die Kranke kann zunächst die Zähne gut fletschen; nach ca. 1 Minute sinkt die Oberlippe allmählich herab und die Kranke will dann das 
Gefühl der Spannung in der Oberlippe haben; ebenso auch, wenn sie einige Zeit lang lacht, was zunächst ganz gut geht.

3. X. Die Ptosis ist seit gestern etwas stärker, sie blieb im Laufe des gestrigen Tages gleich, erschien heute Morgen ausgeprägter und hat gegen Abend noch etwas zngenommen. Die Bewegungen der Bulbi erscheinen jetzt objectiv ziemlich normal, doch bestehen noch die beschriebenen Doppelbilder. Das Erheben des 1. Beines geschielt anfangs mit leidlich guter, jedoch viel geringerer Kraft als rechts; nach 11 maligem Heben ist die Kraft erloschen. Die Kranke erhält von jetzt an jeden 2. Tag $1 \mathrm{mg}$ Strychnin. nitr. subcutan; sie will danach eine Znnahme der Kraft in der Extremität, wo die jeweilige Injection gemacht ist, bemerken; doch ist dies objectiv kaum nachweisbar. Händedruck andauernd mit geringerer Kraft als normal. Dynamometerversuch am 6 . X.:

rechts: $20,20,21,20,19,17,16,16,13,13,10,10,10$, Pause, 20,20 links: $22,25,20,25,24,20,20,18,20,18,15,15$

Kraft des Iliopsoas heute sehr gut.

Die elektrische Untersuchung der Gesichtsnerven und -Huskeln ergiebt jetzt folgendes Resultat (7. X.):

Faradisch. Vom Facialisstamm aus beiderseits bei $80 \mathrm{~mm}$ Rollenabstand erste Zuckung. In den Stirnästen fällt die Zuckung bei Reizung am Arcus zygomat. bei derselben Stromstärke geringer aus, als von den Reizpunkten der unteren Aeste. Bei directer Muskelreizung reagiren in den unteren Aesten bei der gleichen Stromstärke alle Muskeln gut; von den Muskeln des ersten Astes reagiren der Frontalis und Corrugator supercilii entschieden schwächer, besser der Orbicul. oculi; in diesem giebt es beiderseits eine kurze Anfangszuckung mit sich ausschliessendem leichten Vibriren der Muskeln, auch bei einer Stromstärke (Rollenabstand 70), die in allen anderen Muskeln Tetanus hervorruft; selbst bei stärksten Strömen entsteht in den Orbiculares kein Tetanus. Eine deutliche Ermüdbarkeit lässt sich für den elektr. Strom nicht nachweisen.

Galvanisch. Vom Stamm und den einzelnen Aesten des Facialis bei 21/2 M.-A. deutliche KSZ; doch bei derselben Stromstärke schon Andeutung von ASZ. Bei directer Muskelreizung in den oberen Facialismuskeln KSZ bei 2 M.-A., im Orbicul. oculi beiderseits $A S Z$ schon bei $13 / 4$ M.-A., in den übrigen Gesichtsmuskeln dentliche Zuckung erst bei $31 / 2$ M.-A. und Ueberwiegen der Kathode. Legt man die Elektrode auf das herabgesunkene rechte obere Lid, so entsteht ein KSTe mit deutlich träge beginnender Zuckung, so dass das Lid dabei gehoben wird (Prof. Schmidt), im linken Lid ist bei derselben Stromstärke noch keine Zuckung zu erzielen. Sonst ist die Zuckung überall blitzartig.

8. X. Das subjective Befinden der Kranken ist heute wieder weniger gut; Menses heute erwartet, bleiben aus. Puls und Athmung beschleunigt. Ptosis sehr stark; Doppelbilder in allen Theilen des Gesichtsfeldes. Schlucken erschwert, Znnge schwerer beweglich, leichtes Lispeln beim Zählen; Gaumensegel unbeweglich, Reflex fehlt. Aufgefordert zu zählen, kann die Kranke dies zuerst rasch und dentlich; von $10 \mathrm{ab}$ werden die einzelnen Zahlen undeutlich, der Luftmangel macht sich geltend; schliesslich wird nach jeder Zahl eine tiefe Inspiration nöthig, die Articulation wird immer verwaschener; weiter als bis 24 (ein anderes Mal nur 10) ge- 
lingt das Zählen überhaupt nicht. Zugleich hat dann die Kranke ein Gefühl von Spannung im Bereiche der oberen Intercostalmuskeln. - Die Kraft im linken Bein ist heute recht gut.

9. X. Der Zustand hat sich wieder gebessert; die Ermüdung beim Zählen ist nicht mehr so ausgesprochen, Respiration annähernd normal. Die Neigung zur Besserung hält in den nächsten Tagen an.

Am 14. X. wird die Kranke anf ihren Wunsch entlassen, nachdem noch einmal ein genauer Status des Nervensystems aufgenommen war, aus dem kurz das Folgende erwähnt sei:

Pupillen ohne Störung. L. Bulbus frei beweglich, der rechte bleibt beim Blick nach oben deutlich zurück, desgleichen beim Blick nach aussen, hierbei wird er nach unten abgelenkt. Beim Blick nach innen Zurückbleiben geringer, Blick nach unten frei. In der r. Hälfte des Gesichtsfeldes gekreuzte Doppelbilder; in der Mittellinie stehen dieselben über einander; in der 1. Gesichtshälfte gekrenzte, schräg übereinander stehende Doppelbilder, das linke steht höher. - Linke Lidspalte von normaler Weite, rechts Ptosis bis zur Mitte der Pupille; auch beim Versuch, das Auge weit zu öffnen, keine wesentliche Veränderung der Ptosis. Lidschluss ist links schlechter als rechts, es bleibt links ein Spalt von ca. $2 \mathrm{~mm}$, rechts höchstens $1 \mathrm{~mm}$. Bei längerem Lidschluss (15 Sec.) tritt Zittern in den unteren und oberen Augenlidern ein; die Kranke hat dann auch das Gefühl, als ob ihr der Lidschluss schwerer fällt; doch ist objectiv kein Nachlassen der Function, auch nicht bei häufiger Wiederholung zu constatiren.

Stirnrunzeln fast ganz unmöglich. Nasolabialfalten beiderseits ziemlich verstrichen. Die unteren Partien der Wangen erscheinen deutlich abgeflacht, Patientin hat hier bei längerem Sprechen oder Lachen ein unangenehmes Gefïhl der Spannung. Lippenschluss, Pfeifen, Mundöffnen, Zähnefletschen etc. gelingt gut und mit normaler Kraft, dabei keine dentliche Ermüdbarkeit; doch tritt bei langem Heben der Oberlippe fibrilläres Zittern derselben ein, aber erst viel später, als beim Angenschluss in den Lidern.

Kaumusculatur ziemlich kräftig, nicht ermüdbar. Das Gaumensegel wird nur eine Spur gehoben, dabei etwas mehr nach links verzogen; Gaumenreflex ganz schwach. Keine Störung beim Schlucken. Die Zunge wird gerade hervorgestreckt, kann seitlich ausgiebig und ziemlich schnell bewegt werden; dabei keine Ermüdbarkeit. Heben der Zunge vom Boden der Mundhöhle gelingt sehr schlecht; die Kranke vermag auch mit der Zungenspitze nicht den harten Gaumen zu berühren. Das Organ erscheint in toto etwas schlaff und dünn; kein deutliches Zittern.

Sternocleidomastoidei wirken kräftig, zeigen keine Ermüdbarkeit.

Aufrichten aus der Rückenlage ist ohne Zuhülfenahme der Arme nicht möglich. Die Bauchmuskeln spannen sich sehr schlecht an. Auch die Action der langen Rückenmuskeln ziemlich kraftlos. An den oberen Extremitäten sind alle Bewegungen, ausser in den Fingern, mit guter Kraft und ohne Ermüdung ausführbar. Zwar will die Kranke in den Streckern des linken Unterarmes eine Mattigkeit fühlen, doch ist objectiv anch bei 20 facher Wiederholung der Bewegung eine Ermüdung kaum angedentet. Die Streckung der Endphalangen des 1. bis 4. Fingers sowie 
Fingerspreizen beiderseits ganz kraftlos. Beim Händedruck keine Ermüdbarkeit.

An den unteren Extremitäten besteht rechts keine Störung. Das linke Bein wird im Hüftgelenk nur mit geringer Kraft und bis zu einem Winkel von ca. $30^{\circ}$ von der Unterlage erhoben; es kann nur 10 Sec. in dieser Stellung gehalten werden. Aufgefordert, das Bein schnell hintereinander zu erheben, vermag die Kranke dies $14 \mathrm{mal}$, die letzten Male immer kraftloser; dann bedarf sie längerer Panse, bis nene Erhebung möglich. Eine Ermüdbarkeit im Bereiche anderer Muskeln ist nach diesem Versuche nicht nachweisbar. Abduction des Oberschenkels mit normaler Kraft, Adduction ganz aufgehoben. Sonst alle Bewegungen kräftig, vielleicht eine geringe Schwäche in den linken Peronei. - Der Gang ist ziemlich langsam, das linke Bein schleppt etwas nach, doch geht Patientin mehrere Minuten ohne Ermüdung.

Reflexe. Radiusr. rechts schwach, links nicht sicher auszulösen. Tricepsr. und Bicepsr. fehlen beiderseits. Patellarr. links sehr schwach, rechts etwas stärker. Achillesr. beiderseits eben angedentet. Von der Planta pedis weder durch Streichen noch Stechen irgend ein Reflex auszulösen. Bauchr. rechts stärker als links.

Die Sprache ist gut articulirt, die Ermüdbarkeit nicht so ansgesprochen wie oben, doch bei den Zungenbuchstaben dentlich. Beim Zählen znnehmende Atheminsufficienz. Sensibilität ist überall - auch im Trigeminusgebiet intact, auch das Lagegefühl nicht gestört.

Puls mässig beschleunigt.

Fassen wir einmal in kurzen Worten das Wesentlichste dieses wechselnden Krankheitsbildes zusammen, so sehen wir bei einer bis dahin gesunden 24jährigen Frau im Anschluss an einen Katarrh der Respirationswege eine Menge motorischer Ausfallserscheinungen in regelloser Aufeinanderfolge sich entwickeln, die in den verschiedensten Nervengebieten (äussere Augenmuskeln, Zunge, Kau- und Schlingmuskeln, Facialisgebiet, Kehlkopf, Athemmuskeln, Finger, Iliopsoas) im bunten Wechsel auftreten. Die Intensität der einzelnen Lähmungen ist, oft im Laufe ein und desselben Tages, sehr schwankend, wie überhaupt während der 5 monatlichen Krankheitsdauer eine auffallende Neigung zu Remissionen und Verschlimmerungen hervortritt. Zuweilen lässt sich in einzelnen Gebieten (Iliopsoas, Articulation, Athemmuskeln) eine mehr oder minder deutliche Ermüdbarkeit nachweisen, während die elektrische myas thenische Reaction während der von uns beobachteten Verlaufszeit fehlt. Dagegen entwickelt sich gegen Ende der Beobachtung eine leichte Entartungsreaction im $r$. Levator palpebrae.

Man wird zugeben müssen, dass die Diagnose des Falles auf einige Schwierigkeiten stiess. Bei der Unbeständigkeit der Symptome hätte man wohl an eineH y sterie denken können, zumal die Kranke psychischer Beeinflussung nicht unzugänglich erschien. Doch widersprach dem 
der Beginn der Krankheit mit Doppeltsehen und mit Ptosis bei der vorher durchaus nicht neuropathisch veranlagten Frau, sowie das Fehlen von Sensibilitätsstörungen und allen sonstigen hysterischen Stigmata. - Mit einer peripherischen Nervenerkrankung, etwa einer Polyneuritis nach Influenza, bot die Erkrankung nur oberflächliche Aehnlichkeit; es fehlten aber deutliche sensible Reiz- und Lähmungserscheinungen; eine rein motorische Neuritis liess sich wegen des Erhaltenseins der Reflexe nicht annehmen; ausserdem pflegt bei keiner Art von Neuritis ein so wechselndes Verhalten der Lähmungen vorzukommen. Von den Affectionen des centralen Nervensystems hätte vielleicht eine cerebrospinale Lues in Betracht kommen können, besonders die gummöse Meningitis der Hirnbasis, da bei ihrem Verlauf mannigfache und plötzliche Schwankungen beobachtet werden; doch fehlten für eine solche Diagnose sowohl das ätiologische Moment, als auch gewisse häufige Symptome wie Kopfweh, Lähmung der inneren Augenmuskeln, Opticusaffection, Störungen der Sehnenreflexe etc.

Am meisten mussten wohl noch die Beziehungen des Krankheitsbildes zur progressiven Bulbärparalyse in die Augen fallen, besonders im späteren Verlaufe der Krankheit, als eine leichte Entartungsreaction sich nachweisen liess. Indessen beginnt die gewöhnliche Bulbärparalyse bekanntlich nicht an den Augenmuskeln, und andererseits fehlte der stetig fortschreitende Charakter der Lähmung im Krankheitsverlauf, der vielmehr mit seinen oft starken Remissionen die Annahme einer tiefer greifenden ,organischen" Läsion unmöglich machte. Deshalb konnten wir uns auch nicht für das Bestehen schwerer entzündlicher Veränderungen in den Nervenkernen, für eine Polioencephalomyelitis, entscheiden, in deren Verlauf freilich mehrfache Intensitätsschwankungen - auch im Sinne der Ermüdbarkeit - beobachtet wurden, jedoch nicht so ausgeprägte und dauernde Remissionen vorkommen.

Es blieb also nichts übrig als die Annahme einer „Lähmung ohne anatomischen Befund", einer "asthenischen Paralyse" zu machen, wobei von der Entartungsreaction in einem einzelnen Muskelgebiet abgesehen wurde.

Denn mit dieser Annahme stimmten die wesentlichen Züge des Krankheitsbildes überein.

Das gilt zunächst von der Localisation der Lähmungen, wie nicht weiter ausgeführt $\mathrm{zu}$ werden braucht. Bemerkenswerth war in unserem Falle die besonders starke Betheiligung des linken Iliopsoas, ferner diejenige der Rumpfmuskeln und endlich die sonst recht selten beobachtete Lähmung oder Schwäche der Kehlkopfmuskeln, die in unserem Falle Monate lang in fast gleicher Intensität bestand. 
In der Literatur findet sich eine stärkere Betheiligung des Larynx nur in den Fällen von Hoppe $\left.{ }^{1}\right)$ und Kostetzkij2) erwähnt, ausserdem ist noch zuweilen eine Ermüdbarkeit der Stimme beobachtet worden.

Des Weiteren passen der eigenthümliche Krankheitsverlauf unseres Falles, der zeitliche und örtliche Wechsel der Ausfallserscheinungen, sowie die Intensitätsschwankungen gut zum Bilde der „Myasthenie". Bemerkenswerth ist, dass zweimal die Verschlimmerung mit Beginn der Menstruation eintrat und durch die Betheiligung der Athemmuskeln sogar einen bedrohlichen Grad erreichte. Die Remission betrifft in typischer Weise nie alle Schwächeerscheinungen zugleich, sondern stets nur einzelne Symptome, während zur selben Zeit in anderen Muskelgebieten Verschlimmerungen auftreten können.

Weit weniger in den Vordergrund tritt bei unserer Kranken ein anderes Symptom, das in den letzten Jahren, besonders seit den Arbeiten von Goldflam ${ }^{3}$ ) und $\mathrm{J}_{0} \mathrm{lly}^{4}$ ), gradezu als pathognomonisch für die asthenischen Lähmungen gilt und ihnen auch den Namen gegeben hat: die allgemeine Muskelermüdbarkeit. Es ist schwer zu sagen, inwieweit dieselbe bei den älteren Fällen nur, weil sie nicht bekannt war, nicht bemerkt wurde oder wirklich fehlte; dass sie nicht regelmässig da zu sein braucht, beweist gerade wieder eine der jüngsten Veröffentlichungen von "Bulbärparalyse ohne anatomischen Befund" $\left(\right.$ Schüle $\left.{ }^{5}\right)$ ). Gewöhnlich ist auch in ein und demselben Falle die Ermüdbarkeit nicht immer oder in sehr wechselndem Grade und meist nur in einzelnen Muskeln nachzuweisen. So war es auch bei unserer Kranken: anfangs sprachen höchstens die subjectiven Angaben für das Bestehen einer rascheren Ermüdung, und zuweilen war die Ptosis Morgens in geringerem Grade ausgeprägt, als gegen Ende des Tages; später jedoch konnte eine deutliche Erschöpfbarkeit beim Sprechen beobachtet werden, an der auch die Athemmuskeln theilnahmen. Mehrmals ergab auch der Dynamometerversuch ein schnelleres Nachlassen der Kraft in den Händen. Am deutlichsten und häufigsten aber trat die Myasthenie bei der Beugung des Oberschenkels zu Tage. Dabei ist jedoch zu betonen, dass hier stets nur an solchen Tagen die Ermüdbarkeit sich deutlich constatiren liess, an denen schon von vornherein die motorische Kraft erheblich herabgesetzt war, so dass gleich die ersten Bewegungen mangelhaft und kraftlos ausfielen, während in denklassischen Myastheniefällen anfänglich die Bewegungen mit normaler Kraft

1) Berl. klin. Wochenschr. 1892. Nr. 14.

2) Neurol. CentralbI. 1899. S. 546.

3) D. Zeitschr. f. Nervenh. Bd. IV.

4) Berl. klin. Wochenschr. 1895. Nr. 1.

5) Münch. med. Wochenschr. 1899. Nr. 13. 
geschehen, dann aber rasch nachlassen. - Im Bereich der Hirnnerven war zuvor eine schnellere Abnahme der Muskelaction kaum angedeutet, doch machte sich die Ermüdung auffallenderweise dadurch kenntlich, dass nach längerem Augenschluss und nach längerem Heben der Oberlippe fibrilläres Zittern in den betreffenden Muskeln eintrat.

Das von Goldflam, Jolly u. A. erwähnte Symptom der Ausdehnung der Ermüdbarkeit auf alle Muskeln bei längerer Thätigkeit eines einzelnen liess sich bei unserer Kranken nicht nachweisen. Es können eben offenbar in den einzelnen Krankheitsfällen in den verschiedenen Zeiten des Krankheitsverlaufes die verschiedensten Abstufungen und Localisationen der Ermüdungserscheinungen vorkommen.

Vielfach discutirt worden ist über die Bedeutung der echten, persistirenden Lähmungen im Symptombilde der asthenischen Paralyse. Strümpell ${ }^{1}$ ) neigt zu der Ansicht „dass es sich auch hierbei eigentlich nur um den höchsten Grad der Ermüdbarkeit handelt, schon hervorgerufen durch die kleinsten, unvermeidlichen Ansprüche, welche jederzeit an unser Muskelsystem gestellt werden"; während Oppenheim ${ }^{2}$ ) die Auffassung vertritt, dass in einem Theile der Muskeln wirkliche Lähmung besteht, und dass man deshalb mit Unrecht von einer Pseudoparalyse spricht. In unserem Falle könnte man den Umstand, dass gerade in den fortwährend in Thätigkeit begriffenen Muskeln des Auges und der Stimmbänder eine dauernde Lähmung bestand, zu Gunsten der Strümpell'schen. Theorie anführen.

Der Nachweis der elektrischen Ermüdungsreaction (Jolly) gelang bei unserer Kranken trotz vielfacher Prüfung nicht in typischer Weise; nur bei der ersten Untersuchung konnte Dr. Weber ein Nachlassen der Zuckungsstärke für den galvanischen Strom feststellen. Ferner erhielt man bei Reizung der Orbicul, oculi statt einer einfachen Zuckung ein anhaltendes Vibriren der Muskeln, und es liess sich auch mit stärkeren Strömen kein Tetanus erzielen, eine Erscheinung, die schon in dem Falle von Wernicke-Cohn ${ }^{3}$ ) vorhanden war. Allerdings waren in unseren Falle bei dem vorwiegenden Ergriffensein der Gesichtsmuskeln die Verhältnisse dem Nachweis der myasthenischen Reaction nicht günstig. Versuche, wie z. B. im Laquer'schen Falle ${ }^{4}$, bei dem nach 18-19 maliger Hervorrufung des Tetanus sich eine Ermüdung einstellte, waren bei unserer sehr empfindlichen Kranken im Gesicht einfach nicht ausführbar. Wir möchten deshalb um so weniger Werth auf das negative Ergebniss legen, als die Jolly'sche

1) D. Zeitschr. f. Nervenh. VIII. Bd. 1./2. Hft.

2) Ebenda. VI. Bd. S. 406.

3) D. med. Wochenschr. 1897. Nr. 49.

4) Volkmann's Samml. klin. Vortr. Nr. 205. 
Reaction sich bisher nur in einem Bruchtheile der Fälle gefunden hat; ausserdem will sie Feinberg ${ }^{1}$ ) bei einem Falle von progressiver Bulbärparalyse mit organischer Grundlage in typischer Weise beobachtet haben.

Ueberhaupt wird die Beurtheilung aller dieser hier nur kurz angedeuteten Fragen dadurch erschwert, dass Untersuchungen äber das Vorkommen der Ermüdungsphänomene bei anderen Lähmungsformen fast völlig fehlen. Benedikt ${ }^{2}$ ) und Brenner ${ }^{3}$ ) haben schon vor längerer Zeit bei Hemiplegie das Vorkommen von Ermtudbarkeit beschrieben, doch findet sich in der neueren Literatur nichts über diesen Gegenstand. Bei einem eigenthümlichen hereditär-familiären Nervenleiden, das der Eine von uns (Dr. Giese) in dieser Zeitschrift vor Kurzem beschrieben hat, konnten wir eine deutliche Ermüdung der Sprache und der Respirationsthätigkeit nachweisen, in ganz analoger Weise, wie bei unserer Kranken. Auch an die leichte Ermüdbarkeit der Neurastheniker sei hier erinnert; bei Dynamometerversuchen an solchen Kranken konnten wir häufig Zahlenreihen für die rechte Hand erhalten, wie $40,35,35,30,30,25,28,25,20,19$; oder 50,48, 45, 32, 30, 25 - Resultate, die denen bei der "Myasthenie" in nichts nachstehen. $\mathrm{Ob}$ nicht auch bei organischen Erkrankungen der peripherischen Nerven und Muskeln sich die Ermüdbarkeit findet, ist an sich durchaus nicht auszuschliessen; genauere Untersuchungen auf diesem Gebiet wären wünschenswerth.

Was die weitere Symptomatologie der asthenischen Paralyse angeht, so sei noch kurz erwähnt, dass die Reflexe in einzelnen Fällen abgeschwächt waren oder theilweise fehlten; manchmal fand sich auch eine Steigerung, selbst Fussclonus (Goldflam $\left.{ }^{4}\right)$ ); in der Mehrzahl der Beobachtungen überwiegt jedoch das normale Verhalten. Bei unserer Kranken machte sich auch gegen Ende der Beobachtung eine Herabsetzung der Reflexerregbarkeit, besonders an den oberen Extremitäten, geltend. Sensibilitätsstörungen sind, abgesehen von einem Fall von Kosh ewnikoff 5 ), in dem wahrscheinlich Complication mit Hysterie vorlag, nie beobachtet worden; sie fehlten auch bei unserer Kranken dauerud mit Ausnahme der Parästhesien in der rechten Hand. Dasselbe gilt von der Beschaffenheit der Sphinkteren.

War nach Alledem die Diagnose auf eine asthenische Paralyse wohl mit Sicherheit zu stellen, so boten sich doch für die Beurtheilung

1) Neurol. Centralbl. 1900. S. 103.

2) Elektrotherapie. 1868. S. 51 u. S. 210.

3) Untersuchg. u. Beobachtgn. 1869. Bd. II.

4) 1. c.

5) D. Zeitschr. f. Nervenh. IX. Hft. 3 u. 4. 
des Krankheitsbildes neue Schwierigkeiten, als gegen Ende der Beobachtung Zeichen einer beginnenden degenerativen Atrophie in einzelnen Gesichtsmuskeln deutlich wurden, während eine Dünnheit der Zunge gleich von Anfang an bemerkbar war. Derartige Beobachtungen liegen mehrere in der Literatur vor $\left(\mathrm{Erb}^{1}\right)$, Koshewnikoff ${ }^{2}$ ), Laquer ${ }^{3}$ ), , und es ist vielfach über ihre Zugehörigkeit zur Myasthenie gestritten worden. Laquer hat aus seiner Beobachtung gefolgert, „dass die Muskelerschöpfbarkeit nur einen Vorläufer der Muskelatrophie und Muskellähmung darstellt, wenn der Verlauf nicht durch einen plötzlichen Tod abgekürzt wird". Hiergegen spricht entschieden der Ausgang mehrerer Fälle in Heilung und der eminent chronische Verlauf anderer (z. B. Fall Murri ${ }^{4}$ ): Exitus nach 10 Jahren, ohne alle Atrophien etc.). Und warum sollte man nicht annehmen, dass durch ein und dieselbe Ursache in den meisten Nervengebieten zwar nur vorïbergehende leichte Schädigungen bewirkt, in einzelnen Theilen aber dauernde, tiefer greifende luäsionen gesetzt werden? Dass übrigens auch diese degenerativen Zustände nicht irreparabel zu sein brauchen, beweist der Fall Koshewnikoff's, indem sich eine mit EaR einhergehende Zungenatrophie wieder erheblich zurückbildete. - Wenn daher auch zuzugeben ist, dass wir es in unserem Falle nicht gerade mit einer sehr typischen Form der Myasthenie zu thun haben, so konnte doch im Wesentlichen diese Diagnose nicht erschüttert werden.

Damit war auch die Vorhersage des Falles zwar nicht eine absolut ungünstige, doch immerhin recht trübe. Unter einigen 50 Fällen konnte nur $7 \mathrm{mal}$ ein Ausgang in Heilung constatirt werden; bei diesen Kranken machte sich dann meist von vornherein die Neigung zum günstigen Verlauf bemerkbar, während bei unserer Kranken das Leiden allen therapeutischen Versuchen hartnäckigen Widerstand entgegensetzte. Auch musste die Betheiligung der Athemmuskeln als ein recht ungtinstiges Symptom angesehen werden, da in mehr als der Hälfte der Fälle ein plötzlicher Exitus durch Respirationslähmung zu Stande kam.

Der weitere Verlauf der Erkrankung sollte denn auch sehr rasch unseren Befürchtungen Recht geben.

Fortsetzung der Krankengeschichte und Antopsie (Dr. Giese).

Nach der Entlassung aus der Klinik war der Zustand der Kranken zn Hause anfangs unverändert. Den Angehörigen fielen als einzige danernde

1) Archiy f. Psychiatr. IX.

2) D. Zeitschr. f. Nervenh. IX. Hft, 3. u. 4.

3) 1 . c.

4) Neurol. Centralbl. 1899. 
Krankheitssymptome die völlige Aphonie und die Ptosis des r. Angenlides auf. Am Abend des 23. October erfuhr die Kranke eine hochgradige psychische Aufregung durch den Tod ihres Kindes; im Anschluss daran trat eine heftige Verschlimmerung ihres Zustandes auf. Der hinzugerufene Arzt constatirte stärkste Dyspnoe, gesteigerte Pulsfrequenz, völlige Lähmung der Sprach-, Schling- und Kaumusculatur. Nach subcutaner Morphiuminjection liess die Athemnoth etwas nach, wiederholte sich jedoch anfallsweise in der folgenden Nacht mehrfach. In einem solchen Anfall erfolgte am 24. Morgens der Exitus letalis.

Die Autopsie konnte ich erst 30 St. post mortem im Hause der Todten vornehmen und musste sie anf die Eröffnung der Schädelhöhle beschränken. Bezüglich des Sectionsbefundes kann ich mich kurz fassen: Das Schädeldach sehr dick; Hirnhäute, Sinus, Gefässe normal. An der Basis cranii nichts Abnormes. Consistenz des Gehirns und Blutgehalt von mittlerer Beschaffenheit; nirgends Herderkrankungen. Die basalen Nerven bieten keine makroskopischen Verändernngen. Zur mikroskopischen Untersuchung wird der Hirnstamm 1 Woche in Formol, dann 5 Tage in Orth'scher Flüssigkeit gehärtet und in Celloidin eingebettet. Die so erhaltenen Schnitte sind sowohl nach den üblichen Carmin- und Markscheidenfärbungen, als auch mit Anilinfarben (nach Nissl, Toluidinblau) gut färbbar.

Der mikroskopische Befund war ein völlig negativer. Zwar finden sich an Nisslpräparaten leichte Abweichungen der Zellbilder vom Nissl'schen Aequivalentbild, insofern die Nisslkörper oft nicht die regelmässige Anordnung zeigen, sondern zum Theil zu klumpigen Verdichtungen neigen, so dass inan von einer Chromatolyse leichtesten Grades sprechen könnte; doch konnte auf diesen Befund nur wenig Werth gelegt werden, da die Autopsie erst 30 St. post mortem stattfand, und die Schnitte - hauptsächlich mit Rücksicht darauf - eine von den Nissl'schen Regeln abweichende Vorbehandlung erfahren hatten. Immerhin liess sich auch so das Fehlen gröberer Veränderungen mit Sicherheit constatiren: Kern und Kernkörperchen sind in allen Zellen normal, nicht wandständig, die Zellfortsätze sind gut erhalten. Die Zellen des III. und VII. Kerns, in denen am ehesten schwerere Veränderungen zu erwarten waren, unterscheiden sich in nichts von den übrigen Nervenzellen. Derselbe Befund lässt sich an Carminpräparaten erheben: nirgends sind in Zahl, Grösse und Form der Zellen irgendwelche Abweichungen vom normalen Verhalten zn finden. Ebenso wenig lassen sich Degenerationen der Markscheiden oder Axencylinder nachweisen; auch die Nervenstämme an der Hirnbasis verhalten sich durchaus normal. An den Blutgefässen bestehen auch keine einwandsfreien Veränderungen; angedeutet findet sich ein leichtester Grad von Arteriosklerose (geringe Intimaverdickung) an einigen Basalarterien; die intramedullären Gefässdurchschnitte sind sicher normal. Auch die häufiger beschriebenen intramedullären Blutangen werden vermisst.

Unsere Diagnose fand also in dem negativen Ergebniss der makroskopischen und mikroskopischen Untersuchung ihre volle Bestätigung. Besonders verdient noch hervorgehoben zu werden, dass auch in den Kernen und Nervenstämmen des Facialis und Hypoglossus trotz der degenerativen Lähmung keine Veränderungen nachweisbar waren. Dieser 
negative Befund stimmt völlig mit dem Resultat der bisher vorliegenden 12 Autopsien überein. Positive Befunde wurden nur in dem Fall von Vidal-Marinesco ${ }^{1}$ ) erhoben, in dem mit Hülfe der Nisslfärbung leichte Veränderungen an einigen motorischen Ganglienzellen gefunden wurden, sowie von Mayer ${ }^{2}$ ), der Degenerationen der intramedullären Hypoglossus-Wurzeln nachweisen konnte. Zu der Beobachtung der französischen Autoren ist zu bemerken, dass ante mortem einige Tage lang Fieber bestand, das recht wohl für die geringfügigen Zellveränderungen (Chromatolyse) angeschuldigt werden kann; und im Falle Mayer waren die Kerne des Hypoglossus normal und die nur mit Marchifärbung nachweisbaren Degenerationen scheinen recht geringfügig gewesen zu sein und erklären jedenfalls das Krankheitsbild in keiner Weise. Alles in Allem liegen also Befunde, welche das Krankheitsbild befriedigend zu erklären im Stande sind, bisher nicht vor. Besonders fehlt es auch noch an Muskelbefunden in den verschiedenen Stadien der Erkrankung.

\section{Schlussbemerkungen (Schultze).}

Ueber den Namen einer Krankheit zu discntiren, wenn das Krankheitsbild selbst im Wesentlichen festgestellt ist, hat wenig Zweck. Auch dann nicht, wenn die vorhandenen Bezeichnungen nicht völlig zutreffend sind oder etwas Hypothetisches enthalten.

So kann in unserem Falle gegen alle Bezeichnungen, welche das Wort „bulbär" enthalten, eingewendet werden, dass sie die gewöhnlich zugleich vorhandene spinale Localisation der Krankheit vernachlässigen. Indessen wiegt doch gewöhnlich das bulbäre Element vor und: Denominatio fit a potiori. Gegen die Bezeichnung als schwere Myasthenie oder Myasthenia pseudoparalytica kann man sagen, dass es bisher nicht, erwiesen ist, dass die Muskeln und nicht die Nerven der wesentliche Sitz der Erkrankung sind, während wieder andererseits durch diesen Namen die Besonderheit der Affection dentlicher hervortritt und ihre Beziehungen zur Jolly'schen myasthenischen Reaction hervorgehoben werden, gerade so wie die Bezeichnung der Thomsen'schen Krankheit als Myotonie zugleich den Hinweis auf die $\mathrm{Erb}$ 'sche myotonische Reaction enthält.

Unter diesen Umständen scheint mir der bereits andererseits vorgeschlagene Name der Erb'schen Krankheit ein durchaus geeigneter zu sein, wenn auch eine stärkere Vermehrung der Personalnomenclatur in der Neuropathologie nicht erwünscht erscheint. Aber ein solcher

1) Presse médical. April 1897.

2) Neurol. Centralbl. 1894. S. 398. 
nichts präjudicirender Namen kann ja wieder verschwinden, sobald eine das Wesen der Krankheit befriedigende Bezeichnung gefunden ist, gerade so wie es heutzutage zweckmässig erscheint, den vieldeutigen Namen der Landry'schen Paralyse nicht mehr anzuwenden.

Von der bekannten Erb'schen Plexuslähmung oder Erb'schen Lähmung ist der Name der Erb'schen Krankheit leicht zu unterscheiden.

Dass unser Krankheitsbild den letzteren Namen oder irgend einen seiner Synonyme vollauf verdient, geht aus den diagnostischen Erörterungen zur Genüge hervor. Dass während der kurzen Zeit unserer Beobachtung die myasthenische Reaction nicht deutlicher und allgemeiner hervortrat, bew eist natürlich, wie schou ausgeführt wurde, nichts gegen unsere Auffassung, ebensowenig wie die Diagnose auf Thomsen'sche Erkrankung dadurch hinfällig wird, wenn sich zur Zeit der Untersuchung die Erb'sche Wellenbewegung nicht nachweisen lässt, oder wenn bei einer Tetanie nicht alle einzelnen bei ihr beobachteten "Phänomene" gefunden werden können.

Auch die geringe Entwicklung einzelner Muskeln und das vereinzelte Auftreten von trägen Zuckungen bei galvanischer oder faradischer Reizung ist in anderen zweifellosen Fällen der gleichen Erkrankung bereits beobachtet worden, von ausgedebnteren Atrophien ganz abgesehen.

Im Hinblick auf diese interessante Erscheinung entsteht, ähnlich wie in anderen Fällen, die Frage, ob es sich nicht um eine Uebergangsform zur gewöhnlichen atrophischen Bulbärparalyse handelte und ob sich die letztgenannte Krankheit nicht vielleicht bei längerer Dauer der Krankbeit entwickelt hätte.

Bei ihrer Beantwortung ist gerade so wie bei der Beurtheilung von Uebergangsbildern auf histologischem Gebiete die grösste Vorsicht geboten.

Es spricht gegen die Annahme solcher Uebergangsformen vor Allem der Umstand, dass in der enormen Ueberzahl der Fälle von atrophischer Bulbärparalyse und chronischer Polioencephalitis ein Symptomencomplex wie derjenige der Erb'schen Krankheit nicht vorausgeht, wenn auch vielleicht das Einzelsymptom der raschen Ermüdbarkeit in den Anfangsstadien dieser Erkrankungen öfters vorhanden sein mag, als man bisher weiss.

Sodann ist die Vertheilung der Lähmungsgebiete eine andere, es besteht eine viel grössere Sy mmetrie der stets doppelseitigen Affection, und der Fortschritt der Krankheit ist viel gleichmässiger etc.

Umgekehrt folgt nur äusserst selten dem anfänglichen Symptomenbilde der Erb'schen Krankheit eine ausgedehnte Muskelatrophie, während allerdings öfters vereinzelte Muskeln zu mässigen Graden von Volumsverminderung gelangen können. 
Warum soll aber nicht, wie schon angeführt. wurde, die unbekannte Schädlichkeit bei der Erb'schen Krankheit auch einmal die lange Zeit hindurch functionell geschädigten oder nur chronisch veränderten Nerven und Muskeln zu stärkerer Atrophie und selbst zur Entartung bringen? Setzt doch auch das Tetanusgift bekanntlich gelegentlich Lähmung und nicht blos einen Reizungszustand!

Dazu kommt, dass die atrophische Bulbärparalyse und die motorische 'Tabes überhaupt mehr ältere Personen bevorzugt, die Erb'sche Krankheit anscheinend viel mehr die jüngeren.

Immerhin wird völlige Klarheit über die Beziehungen beider Krankheiten zu einander erst dann vorhanden sein, wenn wir über die Ursachen derselben besser Bescheid wissen.

In dieser Beziehung ist bis jetzt nur sicher, dass von irgend welcher angeborenen Anlage zu der Erb'schen Krankheit nicht die Rede sein kaun. Ebenso ist es höchst schwierig anzunehmen, dass in einem normal gebildeten Organismus bei jugendlichen Personen eine derartige Erkrankung sich ohne eine von aussen kommende Veranlassung entwickeln sollte. Es bleibt deswegen kaum etwas Anderes übrig, als irgend eine In toxication anzunehmen, mag nun der giftige Stoff von aussen direct eingeführt oder im Anschlusse an irgend welche Störungen im Organismus erzeugt werden.

In letzterer Beziehung sei an eine ganz andere, aber ebenfalls sehr seltene Anomalie, nämlich an die Cystinurie erinnert, welche nach den bekannten Untersuchungen von Baumann einer bestimmten Art von Darmfäulniss ihren Ursprung verdankt. Diese Darmfäulniss muss man sich wohl durch die Einfuhr ganz bestimmter Agentien, seien es Mikroorganismen oder Fermente, oder sonst welche Stoffe, entstanden denken, die an sich sehr selten sind oder nur unter sehr seltenen sonstigen Nebenumständen bei Einzelnen das krankhafte Symptom der Cystinurie erzeugen.

In unserem Falle wird die Annahme einer Intoxication, ähnlich wie in manchen anderen, dadurch besonders nahegelegt, dass die ersten Krankheitserscheinungen sich in ganz ähnlicher Weise an einen acuten Katarrh der Respirationsorgane anschlossen, wie die Diphtherielähmung an eine Diphtherie, bei welcher ja auch, wie in unserem Falle, Augenmuskellähmungen und Gaumensegellähmung mit Regurgitation der Flüssigkeiten durch die Nase sich einzufinden pflegen. Nur war bei unserer Kranken eine Diphtherie nicht constatirt worden und auch der spätere Verlauf der Erkrankung entsprach nicht dem Bilde der gewöhnlichen Diphtherielähmung.

Welcher Art aber dieses Gift sein mag, ob es etwa nach Art mancher Ptomaine wirkt, oder wie sonst, entzieht sich noch völlig 
unserer Kenntniss. Und ob gar durch derartige Gifte sich eine krankhafte Anhäufung von Ermüdungsstoffen in den Muskeln ausbildet, eine Anhäufung, wie sie Laquer annimmt, oder ob überhaupt sich diese Ermüdungsstoffe in grösserer Menge ausbilden, das entzieht sich um so mehr jeder Erörterung, als wir nicht einmal mit Bestimmtheit wissen, ob wirklich die. Muskeln direct geschädigt werden und nicht in erster Linie die Nervensubstanz.

\section{Literatur über „Myasthenie“.}

Die vollständige Literatur bis zum Jahre $1896 / 97$ findet sich bei Fajersztajn (Neurol. Centralbl. 1896. S. 833) und bei T. Cohn (Deut. med. Wochenschr. 1897. Nr. 49). Seitdem liegen folgende Beobachtungen vor:

Koshewnik off, D. Zeitschr. f. Nerrenh. Bd. IX. 3 u. 4 und Neurol. Centralbl. 1897. S. 756.

Kalischer (2 Fälle), D. Zeitschr. f. Nervenh. X, 3 u. 4.

Brissaud u. Lautzenburg (?) (2 Fälle), Neurol. Centralbl. 97. S. 508.

Sänger (2 Fälle), Neurol. Centralbl. 98. S. 287.

Silbermark, Wien. klin. Rundschau. 96. Nr. 45 u. 46.

Eulenburg, Deut. med. Wochenschr. 98. Nr. 1.

Wheaton, Jahresber. d. Neurol. u. Psych. I. S. 666.

Laquer (2 Fälle), Samml. klin. Vortr. Neue Folge 205.

Mailhouse, Boston. medic. Journ. 12. Mai 98.

Sinkler, Journ. of nerv, and ment. diseas. Vol. 26. Nr. 9.

Punton, Ebenda. (Beide ref. i. d. Medicin d. Gegenw. II. 11.)

Kostetzky, Neurol. Centralbl. 99. S, 546.

Campbell, Brit. med. Journ. 99. S. 534.

Montesano, Neurol. Centralbl. 99. S. 548.

Hoffmann, Ebenda. S. 614.

Senator, Berl. klin. Wochenschr. 99. Nr. 8.

T. Cohn, Neurol. CentralbI. 99. S. 1111.

Sü ffer, Ebenda.

Feinberg, Ebenda. 1900. S. 103.

Schüle, Münch. med. Wochenschr. 99. Nr. 13.

A u topsien liegen vor in den Fällen von Oppenheim, Wilks, Senator(2), Shaw, Strümpell, Hoppe, Eisenlohr, T. Cohn, Mayer, Vidal-Mari. nesco, Silbermark. 